\title{
Acompañar y formar. EI mentoreo como herramienta para reducir la deserción escolar
}

\author{
José Carlos Vázquez Parra ${ }^{1 \mathrm{a}}$ y Pille Kustala ${ }^{2}$
}

Tecnológico de Monterrey, Guadalajara, México ${ }^{12}$

Orcid ID: https://orcid.org/ 0000-0001-9197-78261

Orcid ID: https://orcid.org/ 0000-0003-1319-1769²

\section{Recibido: 27 de noviembre de $2017 \quad$ Aceptado: 01 de marzo de 2018}

\section{Resumen}

Los estudios universitarios no solamente significan para los jóvenes contemporáneos un proceso o etapa de formación profesional, sino también, un momento en sus vidas que les presentan diferentes retos que les exigen desarrollar competencias, tanto disciplinares, como sociales y personales. Lamentablemente, si el joven no consigue superar esta prueba, los problemas que trae consigo esta etapa lo superaran, eslabonándose en situaciones que sencillamente se verá incapaz de afrontar. Por ello, las universidades deben ver por herramientas pedagógicas que no solo vean por el desarrollo académico de sus estudiantes, sino también por la adopción de competencias de afrontamiento que les permitan mejorar como personas. El presente artículo, busca hacer una aproximación al mentoring, como posible herramienta de acompañamiento que de manera indirecta puede reducir la deserción escolar al mejorar la experiencia de aprendizaje de los estudiantes universitarios.

Palabras clave: Acompañamiento, Mentee, Tutoreo, Pedagogía, Educación.

\section{Accompany and train. Mentoring as a tool to reduce school dropouts}

\begin{abstract}
University studies not only mean a process or stage of professional training for young people, but also a moment in their lives that presents them with different challenges that require them to develop disciplinary, social and personal competences. Unfortunately, if the young person does not manage to overcome this test, the problems that this stage brings will overcome him, binding himself in situations that he will simply be unable to face. Therefore, universities must find pedagogical tools that not only see the academic development of their students, but 
also the adoption of coping skills that allow them to improve as people. This article seeks to present an approach to mentoring as a possible accompaniment tool that can indirectly reduce the dropout rate by improving the learning experience of university students.

Key-words: Accompaniment, Mentee, Tutoring, Pedagogy, Education.

\section{Introducción}

El ser docente, trae consigo el desempeñar diferentes roles en el proceso educativo, uno debe aprender a ser educador, consejero, instructor, motivador, entre otros.; pero, aunque todos parezcan diferentes en algunas de sus características, tienden y se encaminan a un objetivo común, la mejora en el aprendizaje de nuestros alumnos.

El educar implica a la totalidad del ser humano y de su contexto, por ello, el proceso de aprendizaje resulta tan complejo y debe abarcar sistemas educativos, modelos pedagógicos, medios de comunicación, técnicas didácticas y de involucramiento. De tal forma, la educación es un proceso de crecimiento y cambio integrador que ayuda a que el joven arribe a la madurez intelectual, emocional e incluso física, fundamentándose en la aceptación de sus habilidades, fortalezas y responsabilidades tanto individuales como sociales (Arbizu, Lobato, \& Del Castillo, 2005).

Por lo anterior, el ser docente es una actividad sumamente trascendente, ya que la relación con los alumnos permite no únicamente ser un participante exclusivo del proceso de enseñanza, sino también, de todas las situaciones que rodean el mismo, sobre todo, cuando la relación con sus alumnos tiende a darse de una forma mucho más personal que el método tradicional educativo.

Así, propuestas de acompañamiento como el tutoreo y el mentoreo han tomado un rol determinante en el desarrollo de los nuevos modelos educativos, en donde el profesor y alumno comparten un espacio más cercano, que les permite construir el desarrollo de competencias de manera más directa, personal y consistente (Soler, 2003). Sin embargo, este tipo de herramientas de acompañamiento también permiten el que se pueda poner atención en cuestiones derivadas al proceso de enseñanza, como la interacción del alumno con su entorno, el impacto de su estado personal o incluso, los factores que pueden llevarlo a dejar su educación. Por lo anterior, este tipo de técnica, permiten que el docente amplíe sus habilidades de atención, dejando su rol exclusivo de educador, para convertirse en guía y compañero del proceso de aprendizaje, con todo lo que ello implica (Sánchez, 2008).

El presente artículo, busca hacer una aproximación al mentoreo, como herramienta de mejoramiento, no solo del aprendizaje o desarrollo de competencias, sino también de la capacidad de afrontamiento de los estudiantes que atraviesan por situaciones que usualmente derivan en deserción escolar. A partir de información de un programa de mentoreo de una universidad privada mexicana, se hará una reflexión sobre diversos casos o situaciones en los que se ha reducido la deserción estudiantil a partir de esta técnica educativa.

\section{EI Mentoreo}


El mentoreo, aunque últimamente ha resonado bastante en los ámbitos educativos, no tiene nada de nuevo, pues a lo largo de la historia, encontramos la figura del mentor, misma a la que se le han encomendado muy diversos roles (Casado, Lezcano, \& Colomer, 2015). Sus antecedentes históricos datan desde la antigua Grecia, en el que los grandes filósofos mentoreaban a jóvenes en su desarrollo. Etimológicamente hablando, el mentoreo proviene de la palabra "mentor" que hace referencia a Mentor, que fue a quien Ulises encomienda la educación de su hijo Telémaco. Según el relato, Mentor era la personificación de Atenea, la Diosa de la Sabiduría, queriendo explicar su relación con el proceso de enseñanza que desempeñaba (Klasen \& Clutterbuck, 2002).

De igual forma, hablar de mentoreo nos lleva inevitablemente a referirnos a la mayéutica Socrática, la cual, mediante el diálogo y la oposición de concepciones, lograba acceder y desarrollar el conocimiento en los jóvenes (Haggard, Doufherty, Turban, \& Wilbank, 2011). El filósofo utiliza los cuestionamientos como un medio continuo de generar dudas y respuestas en sus discípulos sobre su propia vida, considerando que solo en la reflexión, el estudiante consigue sacar a la luz el conocimiento que le permite mejorar como individuo.

Sin embargo, no solo en la antigua Grecia se podía apreciar este proceso de acompañamiento, en la época medieval el tutor-mentor representaba un rol de guardia de la vida de sus pupilos, buscando que los mismos se desenvolvieran de una manera correcta, desarrollando buenas costumbres y ejerciendo óptimamente los principios y esencia de la religión (Girves, Zepeda, \& Gwathmey, 2005). En las Universidades del medievo, la figura de los maestro-mentor va poco a poco apegándose al área académica, aunque en este momento histórico, el mentor seguía atendiendo gran parte de las cuestiones personales que aquejaban a sus estudiantes. Esto se repitió durante el renacimiento en donde la figura de mentor y aprendiz era parte natural del desarrollo de las ciencias y las artes (Hobson, Ashby, Malderez, \& Tomlinson, 2009).

Así, aunque ha ido evolucionando el concepto y noción de los participantes del proceso, el mentoreo ha sido una herramienta fundamental en la formación de nuevos individuos y en el desarrollo de habilidades profesiones (Cuenca, García, \& Aizaguirre, 2016). En la actualidad, la figura más común que tenemos del mentor es aquella que se da en los procesos doctorales, en los cuales asesor y estudiante comparten la responsabilidad del desarrollo de un proyecto de investigación, convirtiéndose el profesor en el guía, supervisor y autoridad que avala la investigación y, por ende, es necesario un trabajo de manera directo y cercano (Hobson, Ashby, Malderez, \& Tomlinson, 2009). Algo que caracteriza al desarrollo de procesos doctorales, a diferencia de otros momentos de enseñanza, es que, en estos, asesor y doctorante trabajan a la par en la consecución de un objetivo, desarrollándose un proceso que se asemeja más a un acompañamiento que a una dirección, pues éste no implica, necesariamente, la relación asimétrica que lleva consigo la relación maestro-alumno.

Lamentablemente, en muchas instituciones educativas, el papel que desempeña el mentor se relaciona directamente con cierta orientación a temas institucionales, confundiendo nociones como el tutoreo y el mentoreo, las cuales, aunque se asemejan, por ser procesos de seguimiento de desarrollo de los estudiantes, no son iguales (Arbizu, Lobato, \& Del Castillo, 2005).

Hay muchas definiciones de mentoreo, sin embargo, una de las más completas, y adecuada para los fines del presente artículo es la de la Sociedad Internacional Misionera: "El mentoreo 
es una relación con el propósito deliberado de promover el crecimiento"”, (ACP, 2011). Independientemente del propósito del mentoreo, sea para acompañar un nuevo empleado en la empresa o a un estudiante en la academia, la acción del mismo involucra siempre ser un guía, un facilitador de crecimiento personal de la otra persona, del mentee (Jiménez \& Lorenzo, 2009). De esta manera, podemos llegar al punto en que se entiende al mentoreo como un proceso de acompañamiento.

Aunque la noción esté en constante evolución, si algo han observado los académicos en cuanto a los procesos de mentoreo son sus efectos, considerando que gracias a esta herramienta la actitud del joven aprendiz o mentee mejora considerablemente, haciéndolo más adaptable y con mayor resiliencia ante los problemas (Washburn, 2010).

\section{El mentor}

Ser docente no es una labor que cualquier profesionista pueda desempeñar, ya que no es lo mismo saber de un tema, que tener las competencias para poder enseñarlo. Sin embargo, esta situación se da de igual manera entre docentes y mentores, ya que, aunque resulta semejante el proceso de formación que ambos realizan, la forma de llevarlo a acabo, implica de características que difieren tanto a nivel profesional, pedagógico, e incluso de personalidad (Alonso, Sánchez, Macías, \& Calles, 2009).

Inicialmente, el mentoreo debe percibirse como un proceso de acompañamiento, no uno de enseñanza, y aunque el mentee efectivamente resulta con aprendizajes, estos se dan según sus necesidades y no según el deseo del mentor (Guerra \& Lobato, 2015). A diferencia de los procesos de enseñanza, el mentor, debe provocar una reestructuración de las habilidades y competencias de aprendizaje en el mentee, dedicando parte del trabajo con el aprendiz a resolver sus intereses personales, descubriendo qué es lo que realmente le interesa y que le gustaría saber (Haggard, Doufherty, Turban, \& Wilbank, 2011).

La relación de mentor-mentee debe buscar desarrollar las potencialidades del individuo, principalmente el pensamiento crítico, capacidad creativa y el desarrollo personal. Así, la mentoría se configura como un intercambio de conocimientos y experiencias entre el mentor y su mentee facilitando de tal forma el proceso de aprendizaje, la adquisición de técnicas y herramientas educativas y el desarrollo de la consciencia del alumno sobre las estrategias que utiliza para aprender (Alonso, Sánchez, \& Calles, 2011). Por ello, es necesario que todos aquellos que son parte de este proceso de formación cuenten con las herramientas necesarias para responder a las necesidades de las instituciones, pero, sobre todo, de los jóvenes aprendices (Casado, Lezcano, \& Colomer, 2015).

Un mentor, debe, además de las características propias de cualquier docente, contar con ciertos rasgos fundamentales que le permiten llevar un mejor proceso de acompañamiento:

- Disponibilidad emocional e interés auténtico, para el involucramiento en el proceso de mejora del otro. Sobre todo, tomando en cuenta la responsabilidad de proveer su tiempo según lo requerido por el mentee, no según los tiempos del mentor (Girves, Zepeda, \& Gwathmey, 2005)

- Gran capacidad de escucha. Y no solo de escucha, que registra intercambio de información, pero de escucha activa, que involucra también saber escuchar el silencio del mentee. Aquí el mentoreo resulta a la primera vista muy similar al coacheo. Sin 
embargo, son procesos diferentes por su proceso y por su resultado. El proceso de coacheo involucra un grado de incondicionalidad, lo que el mentoreo no llega experimentar (Cantera, 2002).

- Discreción y habilidad de crear confianza. Ya que al final de cuentas, gran parte de sus resultados dependen de la relación de acompañamiento que pueda forjar con su mentee, quien, si no se siente cómodo, simplemente no permitirá esta interacción (Hansford, Tennent, \& Ehrich, 2003).

- Un mentor debe de mantener siempre neutral en el proceso del mentoreo, teniendo presente que el mentoreo es un proceso de su mentee, no del mentor mismo. El mentor no debe de involucrar emocionalmente, ni olvidar que las observaciones o consejos deben que ser objetivos, tratar de la mejora del mentee y por lo mismo, a la medida de lo posible, deben no ser subjetivos y nunca ser una entrega de juicios hacia la persona del mentee (Manzano, Martín, Sánchez, Rísquez, \& Suárez, 2012).

- Siempre pone la conexión humana, la relación con su mentee antes del proceso de mentoreo: El éxito del mentoring se basa en una estrecha conexión humana entre las dos partes, en una relación de confianza y por lo tanto enfoca en la persona, no en las tecnicidades del proceso. En el momento, que se empieza enfocar en la duración de las entrevistas o en el cumplimiento de los pasos del proceso, se convierte en el proceso de enseñanza y pierde su característica de acompañamiento (Shea, 1992).

- Enfocarse en la persona del mentee, no en los conocimientos o competencias del mismo. El mentoreo se perfila en las necesidades de la persona del mentee, toma en cuenta también las razones personales, emocionales, más allá de las meramente académicas o laborales (Thorndyke, Gusic, \& Milner, 2008).

- Promueve siempre el mensaje optimista, nunca pesimista: El mentoreo promueve la creación de la autoconfianza e capacidad de autogestión del mentee. Por lo mismo, se enfoca en resaltar lo positivo en el mentee y ver su crecimiento como una constante detección de áreas de oportunidad, donde la persona quien se da cuenta de sus áreas de oportunidad, es el mismo mentee (Kram, 1985).

- Es leal a las necesidades del mentee, no de la empresa: El mentoreo se concluye con el crecimiento, cambio del mentee, hacia el reconocimiento de las fortalezas de el mismo, de sus capacidades de enfrentar asuntos que anteriormente declaraba como problemas, con un enfoque nuevo de enfrentarlos, "tacklearlos", en lugar de rendir ante la imposibilidad de solución. Eso sucede desde la capacidad y necesidad del mentee y tiene que tomar en cuenta su posibilidad de crecimiento, no la necesidad de la empresa o de la academia. Si la empresa o la academia no son idóneos para este crecimiento, entonces el deber del mentor es hacerle saber eso al mentee y apoyarlo en el proceso de encontrar su ambiente idóneo (Oste, André, Kassouf, \& Silva, 2014).

De esta forma, es que se puede señalar que, aunque el mentoreo debe abarcar diferentes campos y acciones, según las directrices que marque cada institución, no debe perder de vista que este proceso es más que una herramienta exclusiva de aprendizaje, constituyéndose como un medio para motivar, analizar, apoyar y evaluar el desarrollo y los logros de los mentee. A diferencia de la pedagogía tradicional, el mentoreo ayuda al joven estudiante a sobrellevar y superar las posibles dificultades que se viven en toda su experiencia de formación, sean éstas académicas, emocionales, sociales o personales (Budge, 2006). 


\section{El mentoreo y su relación con la deserción escolar universitaria}

No cabe duda que la educación profesional o superior viene aparejada con serias dificultades que todo estudiante debe superar, mismas que no se relacionan únicamente con cuestiones educativas, sino también con el momento y entorno en el que el joven estudiante se encuentra (Silva, 2005). Factores como la edad, la familia, la situación económica y el desarrollo de sentimientos y emociones característicos de la juventud como la independencia y la autonomía, hacen que los estudios universitarios sean un periodo lleno de retos (Cabrera, Bethencourt, Álvarez, \& González, 2006). Esto, lógicamente, termina impactando en la capacidad de los estudiantes para llevar a buen término sus estudios.

Según datos de ANUIES, la eficiencia terminal en el año 2000 en México solo alcanzaba el 39\%, lo que es considerablemente bajo, si se toma como referencia que una década antes ésta llegaba a más del 50\% (Vélez \& López, 2004).

Entendemos por deserción escolar al retiro, temporal o definitivo, del sistema educativo, siendo esto una causal de la falta de conclusión de los estudios. Múltiples estudios han intentado encontrar causas o factores que llevan a la incapacidad de las instituciones educativas para retener a sus poblaciones de estudiantes, señalando como uno de los principales, la falta de integración de los jóvenes al sistema universitario (Reyes, Castañeda, \& Pabón, 2012).

Aunque este problema puede relacionarse con otros elementos como la limitada capacidad de estudiar o las problemáticas socioeconómicas o familiares, todo termina en un solo punto, la imposibilidad del joven de poder hacer frente a los problemas en esta etapa de su vida, lo que lo lleva a renunciar ante los imprevistos que terminan superándolo (Espinoza, Castillo, González, \& Loyola, 2012).

Por ello, es que el proceso del mentoreo puede, como lo propone este artículo, ser una excelente herramienta para apoyar a los jóvenes a mejorar su capacidad de afrontamiento, ya que el mentoreo involucra acompañamiento paciente y sin prejuicios, siendo un proceso que se enfoca en darle fe y autoconfianza al mentee, buscando apoyarlo en la búsqueda de soluciones, no solo reconocimiento de la existencia de los problemas (Núñez \& Grande, 2012).

Hay que tener claro que, en la academia, cuando un alumno llega a pedir ayuda para resolver un problema, es porque seguramente éste ya lo ha superado, y por ende necesita apoyo externo. Sin embargo, y lamentablemente, no todos tienen la confianza para aproximarse, y mucho menos, pedir ayuda. Por eso, es que son necesarios los mecanismos para lograr la detección de indicios de este tipo de problemáticas, evitando de esta forma, que se generen problemas derivados, como el cambio de comportamiento en los alumnos, el abuso en algún de sustancias, el aumento en la reprobación o incluso, la renuncia del proyecto escolar por valorar que es demasiado para ellos (Abdala, Castiglione, \& Infante, 2008).

Efectivamente, existen figuras que plantean roles de acompañamiento, como son los directores de carrera de muchas instituciones, los cuales, por su cercanía a los alumnos, consiguen identificar muchas de estas situaciones problemáticas, sin embargo, es tan complejo hacer convivir las actividades propias del puesto con el seguimiento personalizado de todos los alumnos, que no es raro dejar pasar o no ver señales que pueden o no desencadenar problemas. Además, son posiciones que están encargados de 200-300 alumnos 
o hasta más, en las instituciones grandes, así que la oportunidad de notar problemas a tiempo puede ser escaza, a menos que los estudiantes sean los que se aproximen.

Por ello, es que los programas de mentoreo resultar ser una excelente opción ante esta necesidad, ya que a diferencia de los directores de carrera o los profesores-tutores que tienen una doble función, el mentor tiene un rol que de manera directa se enfoca en el acompañamiento personalizado (Viator \& Scandura, 1991). El mentor, dedica su tiempo en acompañar a los alumnos con consejos de mejora continua, tomando en cuenta múltiples enfoques, así como la personalidad de cada uno de los alumnos acompañados, ofreciéndoles un apoyo a su medida, promoviendo de esta forma, un mejoramiento y desarrollo en las diferentes áreas que implica su formación. Con esto no se pretende desacreditar la labor de otras figuras académicas, sino simplemente explicar por qué el mentoreo da más oportunidades de resultados, por ser un agente especialmente dedicado al proceso de acompañamiento.

\section{Caso de estudio}

Como se ha argumentado en el apartado teórico, el mentoreo, como labor de acompañamiento, busca que los alumnos desarrollen competencias de afrontamiento que les permita tener mayor resiliencia ante los problemas, mismos, que, si no son resueltos en su momento, pueden llegar a agrandarse a tal nivel, que superan las posibilidades del joven de resolverlo.

De igual manera, el mentoreo permite que el mentee tenga mayor confianza en su trabajo, así como en sus posibilidades de desempeñarse como un mejor estudiante, mismo que en la mayoría de los casos, puede verse reflejado en el mejoramiento de su desempeño escolar.

Para dar peso a estos argumentos, se hizo una investigación en una población 300 alumnos que participan de un programa de mentoreo en una universidad privada mexicana. Por ser un estudio de tipo longitudinal, se llevó a cabo una comparativa entre los resultados arrojados en 2 años académicos, es decir, 4 semestres (enero-mayo 2015, agosto-diciembre 2015, enero-mayo 2016 y agosto-diciembre 2016), haciendo un contraste entre los periodos en que se dio mentoreo y en los que no.

Como indicadores de análisis, se recabó información de 3,420 materias cursadas en dicho periodo de tiempo por la población seleccionada, considerando 2 datos: Promedio general de los alumnos y numero de materias reprobadas.

La selección de dichos indicadores, se ha basado en la literatura sobre el tema, en la cual se señala que el índice de reprobación es uno de los puntos que reflejan de manera más clara cuando un alumno no ha conseguido adaptarse a los requerimientos que exige la educación superior, o bien, se carecen de elementos para enfrentar situaciones que se viven durante ese periodo de su vida.

Como elementos de inclusión, dichos alumnos tenían que cumplir con ciertos requerimientos, como, por ejemplo; estar al corriente de todos sus procesos académicos y no dar de baja ninguna de las materias durante el semestre.

Los resultados se presentan a continuación (Tabla 1) 
Tabla 01

Resultados de la muestra

\begin{tabular}{|c|c|c|c|c|}
\hline Periodo & Enero-Mayo 2015 & $\begin{array}{c}\text { Agosto-Diciembre } \\
2015 \\
\end{array}$ & Enero-Mayo 2016 & $\begin{array}{c}\text { Agosto-Diciembre } \\
2016 \\
\end{array}$ \\
\hline Materias cursadas & 533 & 650 & 734 & 1503 \\
\hline $\begin{array}{l}\text { Promedio General } \\
\text { de las materias }\end{array}$ & 80.15 & 81.19 & 81.56 & 83.21 \\
\hline $\begin{array}{l}\text { Numero de } \\
\text { materias } \\
\text { reprobadas } \\
(\text { Promedio }-70.0)\end{array}$ & $49(9.19 \%)$ & $60(9.23 \%)$ & $50(6.81 \%)$ & $103(6.85 \%)$ \\
\hline
\end{tabular}

Fuente: propia

Como se puede apreciar en la tabla 01, los resultados son claros. Si se hace una comparación entre los mismos periodos del 2015 y 2016, se puede apreciar una clara mejoría, tanto en los promedios de las materias, como en el índice de reprobación. De enero-mayo 2015 a enero-mayo 2016, los alumnos que formaron parte de un proceso de mentoreo lograron aumentar en $1.41 \%$ el promedio de sus materias, reduciendo en $2.38 \%$ el promedio de reprobación. De igual manera, de agosto-diciembre 2015 a agosto-diciembre del 2016, la población aumentó $2.02 \%$ el promedio general de las materias, reduciendo en un $2.38 \%$ el índice de reprobación.

Un punto que se reconoce es que los datos que aquí se presentan pudieran relacionarse con variables que este estudio no ha considerado, como por ejemplo el género, cuestiones emocionales, el hecho de ser o no foráneo, el semestre, entre otros. Sin embargo, lo que pretende este artículo es simplemente sembrar un antecedente sobre la necesidad de poner atención en los programas de mentoreo como una herramienta que promueve la palpable mejoría de sus participantes.

\section{Consideraciones finales}

Como toda etapa de la vida, el proceso universitario reta a los jóvenes a situaciones que les exige el desarrollo de competencias no solo de carácter disciplinar, sino también de tipo social, emocional y personal, mismas que les permitan madurar y prepararse para el futuro que les espera una vez se gradúen. Sin embargo, y de manera previa a esta "vida universitaria", el joven debe tener la capacidad para adoptar este nuevo rol, mismo que viene acompañado de situaciones que como estudiante de secundaria o bachiller no había tenido necesidad de enfrentar (Contreras, 2007).

Aspectos como la lejanía de la familia, la presión por ser autónomos, la constitución de un grupo social, la búsqueda de una pareja o simplemente el mayor nivel de exigencia académica, pueden ocasionar que el joven se vea envuelto por problemas que, si no consigue resolver o superar, lleguen a eslabonarse, potencializarse y simplemente rebasen su capacidad de afrontamiento (Reyes, Castañeda, \& Pabón, 2012). Si algo es común en la adolescencia y la primera etapa de la juventud, es que la falta de experiencia puede nublar la racionalidad de los individuos al momento de tomar decisiones, haciendo lo que comúnmente conocemos como "una tormenta en un vaso de agua". Por consiguiente, parece ser que la formación universitaria va más allá de la aportación académica, pues en sus aulas, sus pasillos y zonas de convivencia, los jóvenes forjan una personalidad, misma que los hará mejores personas, 
profesionistas y ciudadanos. Por ello, es labor de todo educador el contribuir, de una u otra forma, a esta nueva responsabilidad (Olave, Cisneros, \& Rojas, 2013).

El presente artículo, tenía por objetivo el hacer una aproximación al mentoreo, buscando arrojar luz a la manera en que esta herramienta podría resultar benéfica para reducir la deserción escolar. La relación entre ambos factores resulta en cierto grado simple, ya que el mentoreo, como auxiliar en los procesos pedagógicos, permite acompañar a los estudiantes en el difícil proceso de evolución entre la vida escolar y la formación profesional.

Como elemento de reflexión, adicional al análisis teórico, se ha presentado información de un programa de mentoreo de una universidad privada mexicana, mostrando datos palpables de la mejoría académica que significa un proceso de acompañamiento como el dado en el mentoreo. Así, y como ya se había señalado con anterioridad, se reconoce que aún hay mucho por señalar sobre el tema, sin embargo, se considera que lo presentado en el presente texto resulta valioso por sí mismo y que plantea las bases para análisis más profundos.

En forma de conclusión, y rescatando nuestro rol como académicos que formamos parte de una institución educativa, cabe señalar que cualquier aportación que signifique mejorar la experiencia de aprendizaje de los estudiantes, resulta valiosa, pues la labor de un docente va más allá de la generación de un producto o la prestación de un servicio, como se da en otras profesiones, su responsabilidad es la de formar personas integras, las cuales vivirán y darán cabida al mundo que todos compartiremos el día de mañana. Por ello es que el docente debe ir más allá de la formación tradicional, enseñando menos, pero acompañando más.

\section{Referencias}

Abdala, S., Castiglione, A., \& Infante, L. (2008). La deserción universitaria. Una asignatura pendiente para la gestión institucional. Cuadernos de la Facultad de Humanidades y Ciencias Sociales, 173-191.

ACP. (2011). American Corporate Partners. Obtenido de Mentoring Handbook: http://www.acp-usa.org/mentoring-program/resources/mentoring-handbook

Alonso, M., Sánchez, C., \& Calles, A. (2011). Satisfacción con el mentor. Diferencias por rol y sexo. Revista española de Pedagogía, 69(250), 485-501.

Alonso, M., Sánchez, C., Macías, J., \& Calles, A. (2009). Validación de una escala de evaluación de las competencias del mentor en contextos universitarios. Mentoring \& Coaching: Universidad y Empresa, 153-169.

Arbizu, F., Lobato, C., \& Del Castillo, L. (2005). Algunos modelos de abordaje de la tutoría universitaria. Revista Psicodidáctica, 10(1), 7-22.

Budge, S. (2006). Peer mentoring in Post-Secondary education: Implications for research and practica. Journal of College Regarding and Learning, 37(1), 73-87.

Cabrera, L., Bethencourt, J., Álvarez, P., \& González, M. (2006). El problema del abandono de los estudios universitarios. Relieve, 12(2), 171-202.

Cantera, J. (2002). Sistema de gestión de conocimiento a través de procesos de coaching y mentoring. Revista de Psicología del trabajo y de las organizaciones, 18(2), 303-318. 
Casado, R., Lezcano, F., \& Colomer, J. (2015). Diez pasos clave en el desarrollo de un programa de mentoría universitaria para estudiantes de nuevo ingreso. Revista Electrónica Educare, 19(2), 155-180.

Contreras, D. (2007). Temas educacionales: Una aproximación a la deserción en la educación media. Revista Enfoques: Ciencia Política y Administración Pública, 103-119.

Cuenca, M., García, M., \& Aizaguirre, A. (2016). Reflexión sobre el mentoring com oexperiencia de ocio educativo y social en personas mayores de 65 años. Revista interuniversitaria de formación del profesorado, 30(2), 77-88.

Espinoza, O., Castillo, D., González, L., \& Loyola, J. (2012). Factores familiares asociados a la deserción escolar en Chile. Revista de Ciencias Sociales, XVIII(1), 136-150.

Girves, J., Zepeda, Y., \& Gwathmey, J. (2005). Mentoring in a Post-addifmativa action world. Journal of Social Issues, 61(3), 449-479.

Guerra, N., \& Lobato, C. (2015). Caracteristicas del mentoring en un programa de innovación educativa con profesorado universitario. International Journal of Developmental and Educational Psychology, 1(1), 521-530.

Haggard, D., Doufherty, T., Turban, D., \& Wilbank, J. (2011). Who is a Mentor? A review of envolving definitions and implications for research. Journal of Management, 37(1), 280-304.

Hansford, B., Tennent, L., \& Ehrich, L. (2003). Educational mentoring: is it worth the effort? Education Research and Perspectives, 39(1), 42-75.

Hobson, A., Ashby, P., Malderez, A., \& Tomlinson, P. (2009). Mentoring beginning teachers: What we know and what we dont. Teaching and teacher education, 25(1), 207-216.

Jiménez, S., \& Lorenzo, A. (2009). El mentoring como medio formativo en la educación del entrenador de baloncesto. RICYDE. Revista internacional de ciencias del deporte, 5(15), 35-45.

Klasen, C., \& Clutterbuck, D. (2002). Implementing mentoring schemes: A practical guide to succesful programs. Oxford: Butterworth-Heinemann.

Kram, K. (1985). Mentoring at work. Developmental Relationship in Organizational Life. Glenview: Scorr Foresman.

Manzano, N., Martín, A., Sánchez, M., Rísquez, A., \& Suárez, M. (2012). El rol del mentor en un proceso de mentoría universitaria. Educación XXI, 15(2), 93-118.

Núñez, P., \& Grande, F. (2012). El desarrollo de los recursos humanos a través del mentoring: el caso español. Intangible Capital, 8(1), 61-91.

Olave, G., Cisneros, M., \& Rojas, I. (2013). Deserción universitaria y alfabetización académica. Educación y Educadores, 16(3), 455-471. 
Oste, G., André, C., Kassouf, A., \& Silva, D. (2014). Coaching e Mentoring como instrumento de foco no cliente interno: um estudo regional em sao paulo. Revista Brasileira de Marketing, 13(1), 47-59.

Reyes, L., Castañeda, E., \& Pabón, D. (2012). Causas psicosociales de la deserción universitaria. Revista Logos, Ciencia y Tecnología, 4(1), 164-168.

Sánchez, M. (2008). Asesoramiento en la universidad: poniendo a trabajar la experiencia. Profesorado: Revista de currículum y formación del profesorado, 12(1), 1-19.

Shea, G. (1992). Mentoring: A practical guide. Los Altos: Crisp.

Silva, R. (2005). Deserción. ¿Competitividad o gestión? Revista Lasallista de Investigación, 2(2), 64-69.

Soler, R. (2003). Mentoring: Estrategia de desarrollo de recursos humanos. Barcelona: Gestion2000.

Thorndyke, L., Gusic, M., \& Milner, R. (2008). Functional mentoring: A practical Approach with multilevel Outcomes. Journal of continuing education in the health professions, 28(3), 157-164.

Vélez, A., \& López, D. (2004). Estrategias para vencer la deserción universitaria. Educación y educadores, 177-2003.

Viator, R., \& Scandura, T. (1991). A study of mentor-protege relationship in large public accounting firms. Accounting horizon, 20-30.

Washburn, L. (2010). Rethinking mentoring: Comparing policy and practice in special and general education. Education policy analysis archives, 18, 1-25. 